UFIFT-HEP-02-16

\title{
Continuous Spin Representations of the Poincaré and Super-Poincaré Groups
}

\author{
Lars Brink \\ Department of Theoretical Physics \\ Chalmers University of Technology, \\ S-412 96 Göteborg, Sweden
}

\author{
Abu M. Khan, Pierre Ramond, Xiaozhen Xiong \\ Institute for Fundamental Theory, \\ Department of Physics, University of Florida \\ Gainesville FL 32611, USA
}

\begin{abstract}
We construct Wigner's continuous spin representations of the Poincaré algebra for massless particles in higher dimensions. The states are labeled both by the length of a space-like translation vector and the Dynkin indices of the short little group $S O(d-3)$, where $d$ is the space-time dimension. Continuous spin representations are in one-to-one correspondence with representations of the short little group. We also demonstrate how combinations of the bosonic and fermionic representations form supermultiplets of the super-Poincaré algebra. If the light-cone translations are nilpotent, these representations become finite dimensional, but contain zero or negative norm states, and their supersymmetry algebra contains a central charge in four dimensions.
\end{abstract}




\section{Introduction}

The Poincare group is an essential ingredient of relativistic quantum field theories. Its representations in four space-time dimensions were first studied by E. Wigner [1]. Some of its representations describe quantum states found in local field theory: massless particles of fixed helicity and massive particles with or without spin. In string theory, the "front form" construction of the Poincaré group generators [2, 3] has been particularly useful in describing the 26-dimensional bosonic string 近.

Other representations do not seem to be realized by Nature. One is the tachyon representation with negative mass-squared which appears in theories as indicator of instabilities, for instance in spontaneous symmetry breaking. The others are the "continuous spin representations" (CSR), which describe a massless object with an infinity number of helicities. Wigner himself has argued [5] against their use in Physics since they lead to infinite heat capacity of the vacuum. All attempts to associate these representations with physical systems have failed. Several authors [6, 7] have shown their naive quantization implies either non-locality or a breakdown of causality. More recently, Zoller [8] showed how they could arise from a higher-derivative Lagrangian such as the length of the acceleration. In addition, massless theories with helicities greater than two have special problems [9, 10], unless perhaps for theories with an infinite number of degrees of freedom. Not surprisingly, these representations have been forgotten.

Yet, the idea of an infinite number of massless spins has some attractions. Indeed, Vasiliev [11] among others has argued for the existence of such theories. A particular lure is the zero tension (infinite slope) limit of string theories which suggests (classically) an infinite number of massless states with unbounded helicities.

The object of this paper is to study these representations in higher dimensions, and show that they can be assembled in representations of supersymmetry.

\section{The Poincaré Algebra}

The Poincaré generators satisfy the commutation relations,

$$
\begin{aligned}
{\left[P^{\mu}, P^{\nu}\right] } & =0, \\
{\left[M^{\mu \nu}, P^{\sigma}\right] } & =i\left(\eta^{\mu \sigma} P^{\nu}-\eta^{\nu \sigma} P^{\mu}\right), \\
{\left[M^{\mu \nu}, M^{\alpha \beta}\right] } & =i\left(\eta^{\mu \alpha} M^{\nu \beta}+\eta^{\alpha \nu} M^{\beta \mu}+\eta^{\nu \beta} M^{\mu \alpha}+\eta^{\beta \mu} M^{\alpha \nu}\right),
\end{aligned}
$$

where $\eta_{\mu \nu}=(-1,1, \cdots, 1)$. Its representations are characterized by the values of the Casimir operators which are the squared mass $P_{\mu} P^{\mu}$, and the squares of the Pauli-Lubanski forms built out of the Levi-Civitá symbols. In $d$ space-time dimensions, the Pauli-Lubanski $n$-forms are,

$$
W_{\mu_{1} \cdots \mu_{n}}=\frac{\epsilon_{\mu_{1} \cdots \mu_{n} \mu_{n+1} \cdots \mu_{d}} P^{\mu_{d}} M^{\mu_{n+1} \mu_{n+2}} \cdots M^{\mu_{d-2} \mu_{d-1}}}{\sqrt{n ! 2^{\frac{d-n+1}{2}}\left(\frac{d-n-1}{2}\right)\left(\frac{d-n-1}{2}\right) !}}
$$


where $n=1,3, \cdots,(d-3)$ for $d$ even and $n=0,2, \cdots,(d-3)$ for $d$ odd.

It is convenient to express the Poincaré generators in Dirac's [2] front form, derived long ago for spin in four dimensions by Bacry and Chang [3]. For a particle of mass $m$, the Poincaré generators are expressed in terms of transverse positions and momenta

$$
\left[x^{i}, p^{j}\right]=i \delta^{i j}, i, j=1,2, \ldots, d-2 ; \quad\left[x^{-}, p^{+}\right]=-i,
$$

and

$$
x^{ \pm}=\frac{1}{\sqrt{2}}\left(x^{0} \pm x^{d}\right), \quad p^{ \pm}=\frac{1}{\sqrt{2}}\left(p^{0} \pm p^{d}\right) .
$$

The translations are given by

$$
P^{-}=\frac{p^{i} p^{i}+m^{2}}{2 p^{+}}, \quad P^{+}=p^{+}, \quad P^{i}=p^{i},
$$

where $P^{-}$is the light-cone Hamiltonian. The Lorentz generators break up into those which transform the transverse plane into itself, and those which transform out of that plane, (called "kinematic" and "Hamiltonian", respectively by Dirac). The kinematic generators are given by

$$
M^{+i}=-x^{i} p^{+}, \quad M^{+-}=-x^{-} p^{+}, \quad M^{i j}=x^{i} p^{j}-x^{j} p^{i}+S^{i j},
$$

where $S^{i j}$ obey the $S O(d-2)$ Lie algebra of the transverse little group

$$
\left[S^{i j}, S^{k l}\right]=i\left(\delta^{i k} S^{j l}+\delta^{j l} S^{i k}-\delta^{i l} S^{j k}-\delta^{j k} S^{i l}\right) .
$$

The Hamiltonian-like boosts are

$$
M^{-i}=x^{-} p^{i}-\frac{1}{2}\left\{x^{i}, P^{-}\right\}+\frac{1}{p^{+}}\left(T^{i}-p^{j} S^{i j}\right),
$$

where the $T^{i}$ transform as $S O(d-2)$ vectors

$$
\left[S^{i j}, T^{k}\right]=i \delta^{i k} T^{j}-i \delta^{j k} T^{l},
$$

and satisfy

$$
\left[T^{i}, T^{j}\right]=i m^{2} S^{i j} .
$$

When $m \neq 0, T^{i} / m$ are the generators of $S O(d-1) / S O(d-2)$, which together with $S^{i j}$, complete the massive little group $S O(d-1)$.

When $m=0$, the $T^{i}$ commute with one another, acting as light-cone translations, and the algebra can be satisfied in two ways:

- $T^{i}=0$. This corresponds to the familiar massless representations which describe particles with a finite number of degrees of freedom, realized on states that satisfy

$$
T^{i} \mid p^{+}, p^{i} ;\left(a_{1}, \ldots, a_{r}\right)>=0
$$

where $\left(a_{1}, \ldots, a_{r}\right)$ are the Dynkin labels of $S O(d-2)$ representations and $r$ is the rank of the little group. These label the different helicity states of the massless particle. In four dimensions, the Pauli-Lubanski vector is light-like. 
- $T^{i} \neq 0$. In this case, $T^{i}$ are the $c$-number components of a transverse vector. The states on which the Poincaré algebra is realized are

$$
T^{i}\left|p^{+}, p^{i} ; \xi^{i},\left(a_{1}, \ldots, a_{r}\right)>=\xi^{i}\right| p^{+}, p^{i} ; \xi^{i},\left(a_{1}, \ldots, a_{r}\right)>,
$$

which have additional labels, in the form of a little group vector $\xi^{i}$. There is an important difference from the previous case, since $\left(a_{1}, \ldots, a_{r}\right)$ now labels the $S O(d-3)$ subgroup of the transverse little group $S O(d-2)$. In four dimensions, there is no such group and the states are simply labelled by an additional space-like vector of constant magnitude. These span two distinct representations, called "continuous spin representations" by Wigner in his original work [i]. They are characterized by a space-like Pauli-Lubanski vector, and describe a massless state with an infinite number of integer-spaced helicities.

In the following, we construct these representations in higher dimensions, as well as their supersymmetric generalizations.

\subsection{Continuous Spin Representations}

These representations are characterized by the fact that the light-cone translations $T^{i}$ do not vanish, even though $m=0$. It follows that a finite boost creates an infinite number of integer-spaced helicities. To see this, consider a ket that represents a state with light-like momentum aligned along the $z$ direction. In $(3+1)$-dimensions, a rotation in the transverse plane yields

$$
e^{i \phi M^{12}}\left|p^{+}, p^{i}=0 ; \Xi, \alpha>=\right| p^{+}, p^{i}=0 ; \Xi, \alpha+\phi>,
$$

where $\Xi$ is the length of the transverse light-cone translation vector with components

$$
\xi^{1}=\Xi \cos \alpha, \quad \xi^{2}=\Xi \sin \alpha,
$$

which enables us to set

$$
S^{12}=-i \frac{\partial}{\partial \alpha} .
$$

Since $\left[S^{12}, \xi^{i}\right] \neq 0$ for $i=1,2, S^{12}$ (helicity) is no longer a good quantum number; instead it acts on periodic or anti-periodic functions of the angle $\alpha$

$$
\left|\alpha>=\sum_{\lambda} e^{2 \pi i \lambda \alpha}\right| f_{\lambda}>
$$

where $\lambda$ are either integer-spaced integers or half-odd integers. Another way to see the infinite number of helicities is to apply the infinitesimal boost $M^{-i}$ on any state

$$
M^{-i}\left|p^{+}, p^{i}=0 ; \Xi, \alpha>=\frac{T^{i}}{p^{+}}\right| p^{+}, p^{i}=0 ; \Xi, \alpha>
$$


which produces a state which has picked up plus or minus one unit of helicity. In a finite boost, this action is repeated an infinite amount, resulting in states with possible helicities ranging from minus to plus infinity in integer steps. There are (in four dimensions only) two types of representations, those with all integer (single-valued)and those with all half-odd integer (double-valued) helicities. The square of the Pauli-Lubanski vector (Casimir) is the squared length of the translation vector

$$
W^{\mu} W_{\mu}=\vec{T} \cdot \vec{T}=\Xi^{2} .
$$

As we have stated in the introduction, the CSRs have no obvious physical applications, explicitly in local field theories, but the appearance of an infinite number of states may indicate a connection with non-local theories of extended objects. This motivates their study in more general contexts. We explicitly construct these representations in higher dimensions, then proceed to show that they sustain supersymmetry and build continuous spin representations of supersymmetry. Finally we show that if the light-cone translations are nilpotent, the CSRs become finite-dimensional, but at the expense of negative or zero-norm state, and the supersymmetric generalizations yield central charges for these massless representations.

\subsubsection{Higher Dimensions}

The front-form of the Poincaré generators shows that CSR's in any dimensions, correspond to representations of the Galilean little group

$$
\left[T^{i}, T^{j}\right]=0, \quad\left[S^{i j}, T^{k}\right]=i \delta^{i k} T^{j}-i \delta^{j k} T^{l},
$$

where the $T^{i}$ do not vanish and the $S^{i j}$ generate the light-cone little group $S O(d-2)$. Consider $(4+1)$-dimensions, where the light-cone little group is $S O(3)$. In the frame $p^{-}=\vec{p}=0$, the Pauli-Lubanski two-form is

$$
W_{i j}=\frac{1}{\sqrt{2}} \epsilon_{i j k} T^{k}
$$

so that one Casimir is

$$
W_{i j} W_{i j}=\Xi^{2}
$$

as in the 4-dimensional case. The other Casimir is the Pauli-Lubanski zero-form

$$
W=\frac{1}{\sqrt{2}} \epsilon_{i j k} T^{i} S^{j k},
$$

which is the projection of the generator $S^{j k}$ along $T^{i}$. The vector $T^{i}$ then acts as a "quantization axis", along which $W$ assumes the values

$$
\frac{W}{\sqrt{2} \Xi}=0, \pm \frac{1}{2}, \pm 1, \pm \frac{3}{2}, \cdots
$$


It follows that there are two types of representations corresponding to integer and half-odd integer values of $W / \sqrt{2} \Xi$. For each value of $W / \sqrt{2} \Xi$, there corresponds one infinite dimensional representation. Unlike $(3+1)$ dimensions, there are infinite numbers of CSRs in higher dimensions. The states are no longer characterized by the light-cone little group but by its subgroup orthogonal to $T^{i}$, which we call the short little group, $S O(2)$ in this case. Each CSR is labeled by a value of $S O(2)$ (integer of half-odd integer) as well as by the length of $T^{i}$.

It is straightforward to find the CSR representations in terms of $\mid j, m>$, the eigenstates of $S O(3)$, the full little group. Its states are required to be eigenstates of $\vec{T}$, the $S O(2)$ rotations about it, and of the Casimir $\epsilon_{i j k} T^{i} S^{j k}$. As the action of $T^{i}$ on each $\mid j, m>$ yields a linear combination of $j=j, j \pm 1$ states, its eigenstates are infinite linear combinations of eigenstates of the full little group $S O(3)$. To see this in detail, take the light-cone vector in the $z$ direction, so that $\vec{T}$ is like the tensor operator $T_{0}^{1} \equiv Y_{0}^{1}$,

$$
T_{0}^{1}\left|j, m>=\alpha_{+}^{(j m)}\right| j+1, m>+\alpha_{0}^{(j m)}\left|j, m>+\alpha_{-}^{(j m)}\right| j-1, m>,
$$

where the $\alpha$ 's are proportional to Clebsch-Gordan $(\mathrm{C}-\mathrm{G})$ coefficients

$$
\begin{array}{ll}
\alpha_{0}^{(j m)}=\Xi \sqrt{(j+m)(j-m+1)}, & -j<m<j \\
\alpha_{ \pm}^{(j m)}=\Xi \sqrt{\left(j^{\prime}+m\right)\left(j^{\prime}-m+1\right)} ; & j^{\prime}=j \pm 1, \quad-j^{\prime}<m<j^{\prime} .
\end{array}
$$

The state of the form

$$
\left|F>=\sum_{j=|M|}^{\infty} f_{j}^{(M)}\right| j, M>,
$$

is an eigenstate of the Casimir

$$
\frac{1}{2} \epsilon_{i j k} T^{i} S^{j k}|F>=\Xi M| F>, \quad M=0, \pm \frac{1}{2}, \pm 1, \ldots,
$$

and of $T^{3}$, as long as the coefficients satisfy the recursion relations

$\alpha_{-}^{(|M|+p, M)} f_{|M|+p}^{(M)}+\alpha_{+}^{(|M|+p-2, M)} f_{|M|+p-2}^{(M)}+\left(\alpha_{0}^{(|M|+p-1, M)}-\Xi\right) f_{|M|+p-1}^{(M)}=0$, with $p=2,3, \ldots$ and

$$
\alpha_{-}^{(|M|+1, M)} f_{|M|+1}^{(M)}+\left(\alpha_{0}^{(|M|, M)}-\Xi\right) f_{|M|}^{(M)}=0,
$$

since $f_{|M|-1}^{(M)}=0$. The other states of the CSR are determined by acting the $S O(3)$ raising and lowering operators on $\mid F>$. Unlike the usual case, their action does not terminate, producing an infinite number of $S O(3)$ representations. This is similar to Wigner's CSR which contains an infinite number of integer 
spaced helicities. The difference in higher dimensions is that there is an infinite number of CSRs, each labelled by $M$.

In $(5+1)$-dimension the light-cone little group is $S O(4)$, and there are two Pauli-Lubanski forms

$$
\begin{aligned}
& 1-\text { form : } W_{i}=\frac{1}{\sqrt{2}} \epsilon_{i j k l} T^{j} S^{k l}, \\
& 3-\text { form : } W_{i j k}=\frac{1}{\sqrt{6}} \epsilon_{i j k l} T^{l} .
\end{aligned}
$$

The square of the highest form is $\Xi^{2}$, a general feature in any dimensions. The square of one-form is the other Casimir

$$
W_{i} W_{i}=-\Xi^{2} S_{\perp}^{2},
$$

where $S_{\perp}^{i j}$ are the generators of the $S O(3)$ subgroup of $S O(4)$ perpendicular to $T^{i}$, and $S_{\perp}^{2} \equiv S_{\perp}^{k l} S_{\perp}^{l k}(k, l=1,2,3)$ is its Casimir operator. Thus

$$
W_{i} W_{i}=\Xi^{2} j(j+1), \quad j=0, \frac{1}{2}, 1, \frac{3}{2}, \cdots .
$$

There are an infinite number of distinct CSR, one for each value of $j$. The states of the CSR can be labeled by finite rotations of $S O(3)$ and vector $\vec{T}$ characterized by length, $\Xi$, and three angles,

$$
\mid p^{+}, p^{i}=0 ; \Xi, \Omega_{3} ; j, m>\text {. }
$$

where $m$ ranges from $-j$ to $+j$ in integer steps.

In $(6+1)$-dimension the little group is $S O(5)$ and the Pauli-Lubanski forms are,

$$
\begin{aligned}
& 0-\text { form : } W=\frac{1}{2 \sqrt{2}} \epsilon_{i j k l m} T^{i} S^{j k} S^{l m}, \\
& 2-\text { form }: W_{i j}=\frac{1}{2} \epsilon_{i j k l m} T^{k} S^{l m}, \\
& 4-\text { form : } W_{i j k l}=\frac{1}{2 \sqrt{6}} \epsilon_{i j k l m} T^{m} .
\end{aligned}
$$

The two Casimirs of the short little group $S O(4) \approx S U(2) \times S U(2)$, are given by

$$
W_{(2-\text { form })}^{2}=-\Xi^{2} S_{\perp}^{2},
$$

where here $S_{\perp}^{i j}$ are the generators of $S O(4)$ subgroup of $S O(5)$ which leave $\vec{T}$ invariant, and the zero-form which gives the projection of the vector $\epsilon_{i j k l m} S^{j k} S^{l m}$ along $\vec{T}$. The CSR states, characterized by four angles $\Omega_{4}$, and the $\left(j_{1}, j_{2}\right)$ representations of $S U(2) \times S U(2)$, are of the form

$$
\mid p^{+}, p^{i} ; \Xi, \Omega_{4} ; j_{1}, m_{1} ; j_{2}, m_{2}>
$$


where $-j_{1(2)} \leq m_{1(2)} \leq j_{1(2)}$. There is an infinite number of CSRs, each labeled by $j_{1}$ and $j_{2}$.

The pattern is now clear: the CSRs are labeled by the length of a vector and by the irreps of the short little group $S O(d-3)$ which leaves it invariant. Its Casimirs are directly related to the squares of the Pauli-Lubanski $n$-forms and the zero-form. There are infinitely many such representations, fermionic and bosonic.

The case of eleven space-time dimensions is particularly interesting because of M-theory and supergravity. Its CSR's are labelled by $S O(8)$, with its magic triality property which acts on its vector representation, $\boldsymbol{8}_{v}$, and two inequivalent spinor representations, $\boldsymbol{8}_{s}$ and $\boldsymbol{8}_{s}^{\prime}$.

We conclude this section by writing the general expressions for the Casimir operators of the Poincaré algebra in terms of those of the short little group. The Pauli-Lubanski $n$-form for the light-cone little group $S O(d-2)$ is given by Eq.(位),

$$
W_{i_{1} \cdots i_{n}}=\frac{\epsilon_{i_{1} \cdots i_{n} i_{n+1} \cdots i_{d-2}} T^{i_{d-2}} S^{i_{n+1} i_{n+2}} \cdots S^{i_{d-4} i_{d-3}}}{\sqrt{n ! 2^{\frac{d-n-3}{2}}\left(\frac{d-n-3}{2}\right) !}} .
$$

The Casimir operators are simplest to calculate in a frame where only $T^{i_{d-2}} \neq 0$, and the square of the highest form is the squared length of the vector $\vec{T}$. The Casimir operators for $n \geq 1$ are given by,

- for little group $S O(6)$ and $S O(7)$ :

$$
W_{i_{1} \cdots i_{n}}^{2}=\Xi^{2}\left\{\left(S_{\perp}^{2}\right)^{(d-n-3) / 2}-2\left(S_{\perp}^{(d-n-3)}\right)\right\} ; 5 \geq d-n-2>1 .
$$

- For little group $S O(8)$ and $S O(9)$ :

$$
W_{i_{1} \cdots i_{n}}^{2}=\Xi^{2}\left\{\begin{array}{cl}
\left(S_{\perp}^{2}\right)^{(d-n-3) / 2}-2\left(S_{\perp}^{(d-n-3)}\right) & ; \quad 1<d-n-2<7 \\
-\left(S_{\perp}^{2}\right)^{3}+2 S_{\perp}^{2} S_{\perp}^{4}-2 S_{\perp}^{6} \quad ; \quad d-n=9
\end{array}\right.
$$

- In general for the little group $S O(d)$ :

$$
W_{i_{1} \cdots i_{n}}^{2}=\Xi^{2} \sum_{p=0}^{k}\left(A_{2 p} S_{\perp}^{2 p} S_{\perp}^{2(k-p)}+B_{2 p}\left(S_{\perp}^{2}\right)^{k}+C_{2 p}\left(S_{\perp}^{2}\right)^{p} S_{\perp}^{2(k-p)}\right) ，
$$

where $k=\frac{1}{2}(d-n-3)$, and $A_{2 p}, B_{2 p}$ and $C_{2 p}$ 's are numerical constants. In the above, $S_{\perp}^{i j}$ are the generators of $S O(d-3)$ subgroup of $S O(d-2)$ perpendicular to $T^{i_{d-2}}$ and

$$
S_{\perp}^{m} \equiv S_{\perp}^{i_{1} i_{2}} S_{\perp}^{i_{2} i_{3}} \cdots S_{\perp}^{i_{m-1} i_{m}} S_{\perp}^{i_{m} i_{1}}
$$

In odd dimensions, the extra Casimir operator is provided by the Pauli-Lubanski zero-form

$$
W_{(0)}=\frac{1}{\sqrt{2^{\frac{d-3}{2}}\left(\frac{d-3}{2}\right) !}} \epsilon_{i j k \cdots m n} T^{i} S_{\perp}^{i j} \cdots S_{\perp}^{m n} .
$$


In higher dimensions, the CSR states are labeled by $\Xi$, and the solid angle in $d-3$ dimensions, $\Omega_{d-3}$ which give the length and direction of $\vec{T}$, respectively, as well as by $\left(a_{1}, \cdots, a_{r}\right)$, the Dynkin labels of $S O(d-3)$

$$
\mid p^{+}, p^{i} ; \Xi, \Omega_{d-3} ;\left(a_{1}, \cdots, a_{r}\right)>\text {. }
$$

These differ from the usual massless representations in that they are characterized by a space-like vector, and contain an infinite number of states.

\section{Super-Poincaré Algebra}

\subsection{Super-Charges in Light-Cone Form}

The continuous spin representations can be generalized to include supersymmetry. Indeed, Wigner originally found two CSR's, one single-valued with integer helicities, the other double-valued with half-odd integer helicities. As we shall see, they are related by supersymmetry.

The super-Poincaré algebra includes the spinor generators which satisfy

$$
\begin{aligned}
{\left[Q_{A}, p^{\mu}\right] } & =0 \\
{\left[M^{\mu \nu}, Q_{A}\right] } & =-\frac{1}{2}\left(\Sigma^{\mu \nu} Q\right)_{A}, \\
\left\{Q_{A}, Q_{B}^{\dagger}\right\} & =\left(\gamma^{\mu} p_{\mu} \gamma^{0}\right)_{A B},
\end{aligned}
$$

where $A, B$ are spinor indices. In the above,

$$
\Sigma^{\mu \nu}=-\frac{i}{2}\left[\gamma^{\mu}, \gamma^{\nu}\right]
$$

where the $\gamma$ matrices satisfy the anticommutation relation,

$$
\left\{\gamma^{\mu}, \gamma^{\nu}\right\}=2 \eta^{\mu \nu} ; \quad \mu, \nu=0, \cdots, d .
$$

We first consider these relations in four space-time dimensions. The supercharges do not commute with the Pauli-Lubanski vector, as

$$
\left[W_{\mu}, Q_{A}\right]=\frac{i}{2} p^{\nu}\left(\Sigma_{\mu \nu} \gamma_{5} Q\right)_{A}
$$

using

$$
\frac{1}{2} \epsilon_{\mu \nu \rho \sigma} \Sigma^{\rho \sigma}=-i \Sigma_{\mu \nu} \gamma_{5}
$$

and $\gamma^{5}=-i \gamma^{0} \gamma^{1} \gamma^{2} \gamma^{3}$ is the chirality matrix. The supercharges can be realized linearly using Grassmann variables and their derivatives as [12],

$$
Q_{A}=\partial_{A}+\frac{1}{2}\left(\gamma^{\mu} p_{\mu} \gamma^{0}\right)_{A B} \bar{\theta}^{B}
$$


and its conjugate as

$$
Q_{C}^{\dagger}=\bar{\partial}_{C}+\frac{1}{2}\left(\gamma^{\mu} p_{\mu} \gamma^{0}\right)_{D C} \theta^{D}
$$

where we used

$$
\gamma^{0} \gamma^{\mu \dagger} \gamma^{0}=\gamma^{\mu}
$$

and $\partial_{A}=\frac{\partial}{\partial \theta^{A}}, \bar{\partial}_{A}=\frac{\partial}{\partial \bar{\theta}^{A}}$, and $\bar{\theta}_{A}$ is the complex conjugate of $\theta_{A}$ with $A, \cdots, D$ running over the spinor indices. $\theta, \bar{\theta}, \partial$ and $\bar{\partial}$ are anticommuting Grassmann parameters. We use the light-cone projectors to split the supercharge into twocomponent spinors,

$$
Q=Q_{+}+Q_{-}, \quad \text { where } \quad Q_{ \pm} \equiv \mathcal{P}_{ \pm} Q
$$

with $\mathcal{P}_{ \pm}=\frac{1}{2} \gamma^{\mp} \gamma^{ \pm}$. In the Weyl representation, from Eq.(22-24), we can now read off the various anticommutation relations, namely,

$$
\begin{aligned}
& \left\{Q_{+a}, Q_{+b}^{\dagger}\right\}=\sqrt{2} p^{+} \delta_{a b}, \\
& \left\{Q_{-a}, Q_{-b}^{\dagger}\right\}=\sqrt{2} p^{-} \delta_{a b} \equiv \sqrt{2} \frac{p \bar{p}}{p^{+}} \delta_{a b}, \\
& \left\{Q_{-1}, Q_{+1}^{\dagger}\right\}=-\sqrt{2} \bar{p}, \quad\left\{Q_{+1}, Q_{-1}^{\dagger}\right\}=-\sqrt{2} p, \\
& \left\{Q_{+2}, Q_{-2}^{\dagger}\right\}=\sqrt{2} \bar{p}, \quad\left\{Q_{-2}, Q_{+2}^{\dagger}\right\}=\sqrt{2} p,
\end{aligned}
$$

where $p(\bar{p})=\frac{1}{\sqrt{2}}\left(p^{1} \pm i p^{2}\right)$ and $a, b=1,2$. All other anticommutators being zero. This shows that the supercharge splits up into two disconnected sets of anticommutators, corresponding to the left and right projections $\left(\mathcal{P}_{L, R}=\right.$ $\left.\frac{1}{2}\left(1 \pm \gamma_{5}\right)\right)$

$$
\mathcal{Q}_{ \pm}^{R} \equiv \mathcal{P}_{R} Q_{ \pm}, \quad \mathcal{Q}_{ \pm}^{\mathrm{L}} \equiv \mathcal{P}_{L} Q_{ \pm} .
$$

One could also think of these as representing $N=2$ supersymmetry.

There is further reducibility, indicated by the fact that the supercharge anticommutes with the covariant derivative

$$
\mathcal{D}_{A}=\partial_{A}-\frac{1}{2}\left(\gamma^{\mu} p_{\mu} \gamma^{0}\right)_{A B} \bar{\theta}^{B}
$$

and their hermitian conjugates, which give the following relations,

$$
\mathcal{Q}_{-}^{L}=-\frac{\bar{p}}{p^{+}} \mathcal{Q}_{+}^{L}, \quad \mathcal{Q}_{-}^{R}=\frac{p}{p^{+}} \mathcal{Q}_{+}^{R},
$$

together with their conjugates

$$
\mathcal{Q}_{-}^{L \dagger}=-\frac{p}{p^{+}} \mathcal{Q}_{+}^{L \dagger}, \quad \mathcal{Q}_{-}^{R \dagger}=\frac{\bar{p}}{p^{+}} \mathcal{Q}_{+}^{R \dagger},
$$


without affecting the anticommutation relations. From now on we concentrate on the right-handed projections of the algebra. On (right-handed) superfields, the constraint becomes

$$
\mathcal{P}_{R} \mathcal{D} \Phi=0
$$

and after using mass-shell condition, we are left with only one supercharge, $\mathcal{Q}_{+}$(henceforth we drop the superscript $R$ ). It is expressed in terms of one Grassmann variable $\theta_{3} \equiv \theta$ and its conjugate, as we can drop the $\theta_{4}$ variable altogether since its derivative is now expressed in terms of the derivative with respect to $\theta_{3}$. Hence

$$
\mathcal{Q}_{+}=\frac{\partial}{\partial \theta}+\frac{1}{\sqrt{2}} p^{+} \bar{\theta}, \quad \mathcal{Q}_{+}^{\dagger}=\frac{\partial}{\partial \bar{\theta}}+\frac{1}{\sqrt{2}} p^{+} \theta
$$

Since

$$
\left[M^{+-}, \mathcal{Q}_{ \pm}\right]= \pm \frac{i}{2} \mathcal{Q}_{ \pm}
$$

we must extend the Bacry-Chang representation of $M^{+-}$to

$$
M^{+-}=-x^{-} p^{+}-\frac{i}{2}\left(\theta \frac{\partial}{\partial \theta}+\bar{\theta} \frac{\partial}{\partial \bar{\theta}}\right) \text {. }
$$

In order to satisfy the other commutation relations

$$
\left[M^{+i}, M^{-j}\right]=i \delta^{i j} M^{+-}-i M^{i j}, \quad\left[M^{12}, \mathcal{Q}_{ \pm}\right]=\mp \frac{1}{2} \mathcal{Q}_{ \pm}
$$

the generators $M^{12}$ and $M^{-i}$ now include the $\theta$-dependent terms,

$$
M^{12}=x^{1} p^{2}-x^{2} p^{1}+\widehat{S}^{12},
$$

as well as

$$
M^{-i}=x^{-} p^{i}-\frac{1}{2}\left\{x^{i}, P^{-}\right\}+\frac{1}{p^{+}}\left(T^{i}-p^{j} \widehat{S}^{i j}\right)+i \frac{p^{i}}{2 p^{+}}\left(\theta \frac{\partial}{\partial \theta}+\bar{\theta} \frac{\partial}{\partial \bar{\theta}}\right)
$$

where now

$$
\widehat{S}^{12}=S^{12}+\frac{1}{2}\left(\theta \frac{\partial}{\partial \theta}-\bar{\theta} \frac{\partial}{\partial \bar{\theta}}\right)
$$

The generator $M^{+i}$ remains unchanged. The other relevant commutators in light-cone form are

$$
\left[M^{ \pm i}, \mathcal{Q}_{ \pm}\right]=0
$$

In the frame where $p^{i}=0$, we have

$$
\mathcal{Q}_{-}=0, \quad M^{-i}=T^{i} / p^{+}
$$

so that the commutators

$$
\left[M^{-1}, \mathcal{Q}_{+}\right]=-\frac{i}{\sqrt{2}} \mathcal{Q}_{-} \quad\left[M^{-2}, \mathcal{Q}_{+}\right]=-\frac{1}{\sqrt{2}} \mathcal{Q}_{-}
$$




$$
\left[M^{+1}, \mathcal{Q}_{-}\right]=-\frac{i}{\sqrt{2}} \mathcal{Q}_{+}, \quad\left[M^{+2}, \mathcal{Q}_{-}\right]=\frac{1}{\sqrt{2}} \mathcal{Q}_{+}
$$

imply that

$$
\left[T^{i}, \mathcal{Q}_{+}\right]=0
$$

Since the vector that characterizes the CSRs commutes with the supercharge, we can implement supersymmetry on the continuous spin representations without having to change the supercharges.

Thus in $(3+1)$ dimension, we obtain the unique representation of supersymmetry which contains all integer and half-odd integer helicities. This of course does not alleviate the problems associated with the CSRs.

\subsection{Higher Dimensions}

In higher dimensions, Eqs.(20-24) still hold, but the number and nature of independent Grassmann parameters, i.e., whether these are Dirac, Weyl or Majorana type, depend on the number of space-time dimensions 13. In $d$-dimensions, there are $2^{d / 2}\left(2^{(d-1) / 2}\right)$ complex spinor components for $d$ even(odd). Using the anticommutativity between supercharge and covariant derivative, these numbers can be further reduced by a factor of 2 . Let $a, b$ run over the independent spinor indices, i.e., $a, b=1,2, \cdots, 2^{d / 2-m}\left(2^{(d-1) / 2-m}\right)$, for $d$ even(odd) respectively, where $m$ is the number of independent constraints. Using all reducibility conditions, we can always express the super-Poincaré algebra in the light-cone form,

$$
\begin{aligned}
M^{i j} & =x^{i} p^{j}-x^{j} p^{i}+\widehat{S}^{i j}, \\
M^{+-} & =-x^{-} p^{+}+S^{+-}, \\
M^{-i} & =x^{-} p^{i}-\frac{1}{2}\left\{x^{i}, P^{-}\right\}+\frac{1}{p^{+}}\left(T^{i}-p^{j} \widehat{S}^{i j}\right)-\frac{p^{i}}{p^{+}} S^{+-},
\end{aligned}
$$

where

$$
\begin{aligned}
\widehat{S}^{i j} & =S^{i j}+\left(\frac{1}{2} \theta^{a}\left(\gamma^{i j}\right)_{a b} \partial^{b}+c . c .\right), \\
S^{+-} & =\left(\frac{1}{2} \theta^{a}\left(\gamma^{+-}\right)_{a b} \partial^{b}+\text { c.c. }\right),
\end{aligned}
$$

and $\gamma^{i j}$ and $\gamma^{+-}$are the reduced Dirac submatrices consistent with the constraints. $M^{+i}$ remain the same as before. The indices $i, j$ run over transverse space. The supercharges can be irreducibly expressed as,

$$
\mathcal{Q}_{+}^{a}=\frac{\partial}{\partial \theta^{a}}+\frac{1}{\sqrt{2}} p^{+} \bar{\theta}^{a}, \quad \mathcal{Q}_{+}^{\dagger a}=\frac{\partial}{\partial \bar{\theta}^{a}}+\frac{1}{\sqrt{2}} p^{+} \theta^{a},
$$

and $\mathcal{Q}_{-}^{a}$ can be expressed in terms of $\mathcal{Q}_{+}^{a}$. To illustrate this construction, we now show how supersymmetric CSRs arise in five and eleven dimensions: 


\subsection{1 (4+1)-dimensions}

In five dimensions, the spinors have four complex components which can be reduced to two using the covariant derivative constraint. Using the light-cone projectors, $\mathcal{P}_{ \pm}=-\frac{1}{2} \gamma^{\mp} \gamma^{ \pm}$in the representation,

$$
\gamma^{0}=i \sigma^{1} \otimes I, \gamma^{i}=\sigma^{3} \otimes \sigma^{i}, \mathrm{i}=1,2,3 ; \text { and } \gamma^{4}=\sigma^{2} \otimes I,
$$

and Eq.(22), we find

$$
\begin{aligned}
& \left\{\mathcal{Q}_{+a}, \mathcal{Q}_{+b}^{\dagger}\right\}=\sqrt{2} p^{+} \delta_{a b}, \\
& \left\{\mathcal{Q}_{-a}, \mathcal{Q}_{-b}^{\dagger}\right\}=\sqrt{2} p^{-} \delta_{a b}=\frac{\vec{p} \cdot \vec{p}}{\sqrt{2} p^{+}} \delta_{a b}, \\
& \left\{\mathcal{Q}_{+a}, \mathcal{Q}_{-b}^{\dagger}\right\}=i(\vec{\sigma} \cdot \vec{p})_{a b}, \\
& \left\{\mathcal{Q}_{-a}, \mathcal{Q}_{+b}^{\dagger}\right\}=-i(\vec{\sigma} \cdot \vec{p})_{a b},
\end{aligned}
$$

where $a, b=1,2$ and also we used the on-shell condition $p^{-}=p^{i} p^{i} / 2 p^{+}$. From Eq.(26) and $\mathcal{D} \Phi=0$, we find,

$$
\frac{\partial}{\partial \theta^{2+a}}=-i\left(\frac{\vec{\sigma} \cdot \vec{p}}{\sqrt{2} p^{+}}\right)_{a b} \frac{\partial}{\partial \theta^{b}}
$$

and similarly for their complex conjugates, allowing us to set $\left(\theta^{3}, \theta^{4}\right)=0$. The supercharges, Eq.(23.24), reduce to the irreducible form as in Eq.(47) with $a, b=1,2$, and

$$
Q_{-a}=-i\left(\frac{\vec{\sigma} \cdot \vec{p}}{\sqrt{2} p^{+}} Q_{+}\right)_{a} \text { and hence } Q_{-a}^{\dagger}=i\left(Q_{+}^{\dagger} \frac{\vec{\sigma} \cdot \vec{p}}{\sqrt{2} p^{+}}\right)_{a} .
$$

The Lorentz generators remain the same as in Eqs.(42-44) with the following irreducible representations of $S^{i j}$ and $S^{+-}$(in Eq. (45.46)),

$$
\begin{aligned}
S^{+-} & =-\frac{i}{2}\left(\theta \frac{\partial}{\partial \theta}+\bar{\theta} \frac{\partial}{\partial \bar{\theta}}\right), \\
\widehat{S}^{i j} & =S^{i j}+\frac{1}{2} \epsilon^{i j k}\left(\theta \sigma^{k} \frac{\partial}{\partial \theta}+\text { c.c. }\right),
\end{aligned}
$$

where $\theta=\left(\theta^{1}, \theta^{2}\right)$ and $\frac{\partial}{\partial \theta}=\left(\frac{\partial}{\partial \theta^{1}}, \frac{\partial}{\partial \theta^{2}}\right)$. The supercharges transform as $S O(3)$ spinors,

$$
\left[M^{i j}, \mathcal{Q}_{+a}\right]=-\frac{1}{2} \epsilon^{i j k}\left(\sigma^{k} \mathcal{Q}_{+}\right)_{a} .
$$

These charges can be made to act on the CSRs, for which the relevant group is that of the short little group $S O(2)$ which leaves the light-cone translation vector invariant. Aligning $\vec{T}$ along the z-axis, the supercharges split into two 
components, $\mathcal{Q}_{+1}$ and $\mathcal{Q}_{+2}^{\dagger}$, that lower the value of $M^{12}$ by half a unit, and two components, $\mathcal{Q}_{+2}$ and $\mathcal{Q}_{+1}^{\dagger}$ that raise it.

In terms of $M$, the eigenvalue of $\widehat{S}^{12}$, the supermultiplet consists of

$$
\begin{aligned}
\mid M>_{\mathrm{CSR}} & \sim \mid M>_{\mathrm{CSR}}, \\
\mathcal{Q}_{+1}\left|M>_{\mathrm{CSR}}, \mathcal{Q}_{+2}^{\dagger}\right| M>_{\mathrm{CSR}} & \sim \mid M-\frac{1}{2}>_{\mathrm{CSR}}, \\
\mathcal{Q}_{+1} \mathcal{Q}_{+2}^{\dagger} \mid M>_{\mathrm{CSR}} & \sim \mid M-1>_{\mathrm{CSR}}
\end{aligned}
$$

It contains two bosonic and two fermionic CSR, with the same structure as the ordinary $(\vec{T}=0)$ massless $N=2$ supermultiplet in four dimensions. The important difference is that it contains not only the ordinary states but their copies under the boosts proportional to $\vec{T}$. This yields as usual an infinite number of $S O(3)$ polarization states. The action of supersymmetry is the same as in the normal case, but the CSR supermultiplets contain an infinite number of ordinary massless supermultiplets of ever-increasing spin.

\subsection{2 (10+1)-dimensions}

The case of eleven dimensions is particularly interesting because it is shrouded in mystery, as it contains not only local supergravity but also the elusive Mtheory. What we know of M-theory is that its compactifications to lower dimensions yields supersymmetric theories and that its long distance limit is the supergravity theory.

In eleven dimensions, the spinors have 32 complex components, which upon using the Majorana condition and the covariant derivative constraint, reduce to 16 real components. The supercharge splits as

$$
Q=\left(\begin{array}{c}
Q^{A} \\
Q^{16+A}
\end{array}\right) \equiv\left(\begin{array}{c}
Q_{+}^{A} \\
Q_{-}^{A}
\end{array}\right), \quad A=1, \cdots, 16
$$

The Majorana condition implies,

$$
Q_{+}^{A}=Q_{+}^{\dagger A}, \text { and } Q_{-}^{A}=-Q_{-}^{\dagger A} .
$$

In the representation

$$
\gamma^{0}=i \sigma^{1} \otimes I, \quad \gamma^{i}=\sigma^{3} \otimes \tilde{\gamma}^{i}, \quad \gamma^{10}=\sigma^{2} \otimes I,
$$

where $i=1, \cdots, 9$ and $\tilde{\gamma}^{i}$ s are $16 \times 16$ and real, symmetric matrices which satisfy the following algebra

$$
\left\{\tilde{\gamma}^{i}, \tilde{\gamma}^{j}\right\}=2 \delta^{i j}
$$

the super-Poincaré algebra becomes

$$
\left\{Q_{+}^{A}, Q_{+}^{B}\right\}=\sqrt{2} p^{+} \delta^{A B},
$$




$$
\begin{aligned}
\left\{Q_{-}^{A}, Q_{-}^{B}\right\} & =-\sqrt{2} p^{-} \delta^{A B}=-\frac{\vec{p} \cdot \vec{p}}{\sqrt{2} p^{+}} \delta^{A B} \\
\left\{Q_{+}^{A}, Q_{-}^{B}\right\} & =-i \tilde{\gamma}_{i}^{A B} p^{i}, \\
{\left[M^{i j}, Q_{ \pm}^{A}\right] } & =\frac{i}{2}\left(\tilde{\gamma}_{i j} Q_{ \pm}\right)^{A}, \\
{\left[M^{+-}, Q_{ \pm}^{A}\right] } & = \pm \frac{i}{2} Q_{ \pm}^{A}, \\
{\left[M^{ \pm i}, Q_{ \pm}^{A}\right] } & =0, \quad\left[M^{ \pm i}, Q_{\mp}^{A}\right]= \pm \frac{i}{\sqrt{2}}\left(\tilde{\gamma}_{i} Q_{ \pm}\right)^{A} .
\end{aligned}
$$

Using $\mathcal{D} \Phi=0$ and Eq.(26), we find,

$$
\frac{\partial}{\partial \theta^{16+A}}=-i\left(\frac{\tilde{\gamma}^{i} p^{i}}{\sqrt{2} p^{+}} \frac{\partial}{\partial \theta}\right)_{A}
$$

allowing us to set $\theta^{16+A}=0$ for $A=1, \cdots, 16$. The remaining real $Q_{+}^{A}$ 's can be expressed in terms of 16 real Grassmann parameters,

$$
Q_{+}^{A}=\frac{\partial}{\partial \theta^{A}}+\frac{1}{\sqrt{2}} p^{+} \theta^{A} \quad \text { and } \quad Q_{-}^{A}=-i\left(\frac{\tilde{\gamma}^{i} p^{i}}{\sqrt{2} p^{+}} Q_{+}\right)^{A}
$$

Similarly, in this representation, Eqs. $(45-46)$ reduce to

$$
\begin{aligned}
S^{+-} & =-\frac{i}{2} \theta^{A} \frac{\partial}{\partial \theta^{A}}, \\
\widehat{S}^{i j} & =S^{i j}-\frac{i}{2} \theta^{A} \tilde{\gamma}_{A B}^{i j} \frac{\partial}{\partial \theta^{B}},
\end{aligned}
$$

which together with Eqs.(42-44) give the Lorentz generators, where $\tilde{\gamma}^{i j}=\tilde{\gamma}^{i} \tilde{\gamma}^{j}$ for $i \neq j$.

We can let these charges act onto CSRs, remembering that they are labeled by the short little group $S O(8)$ that leaves the light-cone translation vector $\vec{T}$ invariant. We decompose the supercharges into two 8-component supercharge, $\mathcal{Q}_{+}^{a}$ and $\mathcal{Q}_{+}^{\dot{a}}$, by the $S O(8)$ chirality matrix,

$$
Q_{+}^{A}=\left(\begin{array}{c}
\mathcal{Q}_{+}^{a} \\
\mathcal{Q}_{+}^{a}
\end{array}\right), \quad a=1, \cdots, 8
$$

where

$$
\mathcal{Q}_{+}^{a}=\frac{1}{2}\left(1+\tilde{\gamma}^{(9)}\right) \quad \text { and } \quad \mathcal{Q}_{+}^{\dot{a}}=\frac{1}{2}\left(1-\tilde{\gamma}^{(9)}\right),
$$

and the chirality matrix of $S O(8)$ subgroup is

$$
\tilde{\gamma}^{(9)} \equiv \tilde{\gamma}^{1} \tilde{\gamma}^{2} \tilde{\gamma}^{3} \tilde{\gamma}^{4} \tilde{\gamma}^{5} \tilde{\gamma}^{6} \tilde{\gamma}^{7} \tilde{\gamma}^{8}
$$


These two 8-component supercharges furnish two inequivalent spinor representations of $S O(8), \mathbf{8}_{s}$ and $\mathbf{8}_{s}^{\prime}$ characterized by opposite $S O(8)$ chirality and $\mathcal{Q}_{+}^{a} \sim 8_{s}, \mathcal{Q}_{+}^{\dot{a}} \sim 8_{s}^{\prime}$.

The $(16 \times 16) \tilde{\gamma}^{i}$ matrices can be written in terms of $8 \times 8$ block form

$$
\tilde{\gamma}^{i}=\left(\begin{array}{cc}
0 & \tilde{\gamma}_{a \dot{a}}^{i} \\
\tilde{\gamma}_{\dot{b} b}^{i} & 0
\end{array}\right)
$$

where $\tilde{\gamma}_{a \dot{a}}^{i}$ is the transpose of $\tilde{\gamma}_{\dot{a} a}^{i}$. The Clifford algebra for $\tilde{\gamma}^{i}$ is satisfied if

$$
\tilde{\gamma}_{a \dot{a}}^{i} \tilde{\gamma}_{\dot{a} b}^{j}+\tilde{\gamma}_{a \dot{a}}^{j} \tilde{\gamma}_{\dot{a} b}^{i}=2 \delta^{i j} \delta_{a b}, \quad \text { for } i, j, a, b=1, \cdots, 8
$$

and similarly with undotted and dotted indices interchanged. We also define

$$
\tilde{\gamma}_{a b}^{i j}=\frac{1}{2}\left(\tilde{\gamma}_{a \dot{a}}^{i} \tilde{\gamma}_{\dot{a} b}^{j}-\tilde{\gamma}_{a \dot{a}}^{j} \tilde{\gamma}_{\dot{a} b}^{i}\right)
$$

and similarly for $\tilde{\gamma}_{\dot{a} \dot{b}}^{i j}$. The $(8 \times 8) \tilde{\gamma}_{a \dot{a}}^{i}$ matrices couple the vector and spinor representations. In this chiral subspace, the supercharges, $\widehat{S}^{i j}$ and $S^{+-}$take the following irreducible forms

$$
\begin{aligned}
\mathcal{Q}_{+}^{a} & =\frac{\partial}{\partial \theta^{a}}+\frac{1}{\sqrt{2}} p^{+} \theta^{a}, \\
\mathcal{Q}_{+}^{\dot{a}} & =\frac{\partial}{\partial \theta^{\dot{a}}}+\frac{1}{\sqrt{2}} p^{+} \theta^{\dot{a}}, \\
\mathcal{Q}_{-}^{a} & =-\frac{i p^{i}}{\sqrt{2} p^{+}} \tilde{\gamma}_{a \dot{a}}^{i} \mathcal{Q}_{+}^{\dot{a}}, \\
\mathcal{Q}_{-}^{\dot{a}} & =-\frac{i p^{i}}{\sqrt{2} p^{+}} \tilde{\gamma}_{\dot{a} a}^{i} \mathcal{Q}_{+}^{a},
\end{aligned}
$$

as well as

$$
S^{+-}=-\frac{i}{2} \theta^{a} \frac{\partial}{\partial \theta^{a}}, \quad \widehat{S}^{i j}=S^{i j}-\frac{i}{2} \theta^{a} \tilde{\gamma}_{a b}^{i j} \frac{\partial}{\partial \theta^{b}} .
$$

We have two supersymmetries, each transforming as a different $S O(8)$ spinor, and the basic supermultiplet is of the form $\left(\mathbf{8}_{v}+\mathbf{8}_{s}\right)_{\mathrm{CSR}} \times\left(\mathbf{8}_{v}+\mathbf{8}_{s}^{\prime}\right)_{\mathrm{CSR}}$, with 128 bosonic and 128 fermionic states,

$$
\begin{aligned}
& \left(\boldsymbol{8}_{v}+\mathbf{8}_{s}\right)_{\mathrm{CSR}} \times\left(\boldsymbol{8}_{v}+\mathbf{8}_{s}^{\prime}\right)_{\mathrm{CSR}} \\
= & (\mathbf{1}+\mathbf{2 8}+\mathbf{3 5}+\mathbf{8}+\mathbf{5 6})_{v, \mathrm{CSR}}+\left(\mathbf{8}^{\prime}+\mathbf{5} \mathbf{6}^{\prime}+\mathbf{8}+\mathbf{5 6}\right)_{s, \mathrm{CSR}} .
\end{aligned}
$$

This supermultiplet (without CSR) is of course that of the massless states of IIA string theory, obtained by dimensional reduction from eleven-dimensional supergravity.

As we found in the five-dimensional case, there is a one-to-one correspondence between the labels of the ordinary massless representation for $\mathrm{N}=2 \mathrm{su}$ persymmetry in ten dimensions and those of the massless CSR for $\mathrm{N}=1$ supersymmetry in eleven dimensions. However the CSR supermultiplet contains the states of $N=1$ supergravity as well as an infinite number of massless supermultiplets obtained by boosting along $\vec{T}$. 


\subsection{Dimensional Reduction}

The characteristic feature of the CSRs is the transverse space vector $T^{i}$. A non-zero value implies an infinite number of polarization states while a zero value requires a finite number of polarizations. In covariant terms, this vector is written in terms of the Pauli-Lubanski $(d-3)$-form, its magnitude unchanged by Poincaré transformations.

This suggests several ways in which CSRs might play a role in Physics. One is to enlarge the invariance group to include transformations capable of changing the length. The other is to view the CSR in the context of dimensional reduction by limiting transformations to rotations perpendicular to $\vec{T}$.

The former approach requires a study of the representations of the larger groups. Of prime interest are the conformal group (since we are dealing with massless representations) and perhaps the de Sitter groups. In particular we need to identify how CSRs and normal massless representations reside in unitary irreducible representations of these groups.

In physical terms we may view the length as an order parameter (as in the Higgs mechanism with a vector representation). For this we need to give it a dynamical meaning and couple it to an external field; it is amusing to note that in eleven dimensions it naturally couples to an eight-form, which is dual to a 3 -form.

As we let the length tend to zero, we cannot a priori determine which normal representation will be singled out in the transition. This is reminiscent of Dashen's theorem in chiral theories where an external additional tag is required; otherwise it points to a first-order phase transition.

In the context of dimensional reduction, the translation vector $\vec{T}$ naturally singles out a subspace perpendicular to its direction. It follows that group operations restricted to that subspace span normal representations of the Poincaré and super-Poincaré algebras. Thus it might be possible to start with a problematic theory and dimensionally reduce it to a well-defined one.

\subsection{Nilpotent Light-Cone Translations}

We have seen that CSRs necessarily contain massless states of unbounded spins related by finite boosts, raising many objections for their use in the description of point-like objects. On the other hand, the extension of Poincaré invariance to supersymmetry introduces nilpotent Grassmann variables, which suggests the construction of the light-cone translations out of these Grassmann parameters. The translations would be nilpotent, and therefore generate a finite number of helicities with finite boosts.

In $(3+1)$-dimensions, we need two complex Grassmann variables, $\theta_{1}, \theta_{2}$, in order to build an $S O(2)$ vector. We set

$$
T^{1}+i T^{2}=\sqrt{2} \bar{z} p^{+} \theta_{1} \theta_{2}, \quad T^{1}-i T^{2}=\sqrt{2} z p^{+} \bar{\theta}_{1} \bar{\theta}_{2},
$$

where, $z$ is a complex variable, and we have added the appropriate power of $p^{+}$ to ensure proper commutation with $M^{+-} . S^{12}$ now becomes 


$$
\widehat{S}^{12} \equiv S^{12}+\frac{1}{2}\left(\theta^{a} \partial_{a}-\bar{\theta}^{a} \bar{\partial}_{a}\right)
$$

and the Poincaré Casimir operator is now a nilpotent Grassmann number,

$$
W^{2}=W^{\mu} W_{\mu}=-2|z|^{2}\left(p^{+}\right)^{2} \theta_{1} \theta_{2} \bar{\theta}_{1} \bar{\theta}_{2}
$$

With two Grassmann variables, we can construct two supercharges, corresponding to $N=2$ supersymmetry. The kinematic supersymmetries are unaltered

$$
\mathcal{Q}_{+}^{a}=\frac{\partial}{\partial \theta_{a}}+\frac{1}{\sqrt{2}} p^{+} \bar{\theta}_{a} ; \quad \mathcal{Q}_{+}^{a \dagger}=\frac{\partial}{\partial \bar{\theta}_{a}}+\frac{1}{\sqrt{2}} p^{+} \theta_{a}
$$

where $a=1,2$. However the light-cone translations no longer commute with these supercharges. To restore the super-Poincaré algebra, the dynamic supersymmetries must be altered to the new form

$$
\mathcal{Q}_{-}^{a}=\frac{p}{p^{+}} \mathcal{Q}_{+}^{a}-i \bar{z} \epsilon^{a b} \theta_{b}, \quad \mathcal{Q}_{-}^{a \dagger}=\frac{\bar{p}}{p^{+}} \mathcal{Q}_{+}^{a \dagger}+i z \epsilon^{a b} \bar{\theta}_{b}
$$

which ensures the commutation relations of $\mathcal{Q}_{+}^{a}$ with the boosts $M^{-i}$. The resulting supersymmetry algebra

$$
\left\{\mathcal{Q}_{+}^{a}, \mathcal{Q}_{-}^{b}\right\}=i \epsilon^{a b} \bar{z}, \quad\left\{\mathcal{Q}_{+}^{a \dagger}, \mathcal{Q}_{-}^{b \dagger}\right\}=-i \epsilon^{a b} z
$$

acquires central charges, even though we are in a massless representation.

This leads to zero or negative norm states. The positive norm states are of the form $\mid \cdots>$, and $\mathcal{Q}_{+}^{a \dagger} \mid \cdots>$, and $\mathcal{Q}_{+}^{1 \dagger} \mathcal{Q}_{+}^{2 \dagger} \mid \cdots>$, where $\mid \cdots>$ is annihilated by $\mathcal{Q}_{+}^{a}$. However with central charges, in the frame where only $p^{+} \neq 0$,

$$
<\cdots\left|\mathcal{Q}_{+}^{1} \mathcal{Q}_{-}^{2}\right| \cdots>=i z<\cdots \mid \cdots>
$$

and a similar argument with 1,2 interchanged, which shows that the states $\mathcal{Q}_{-}^{a} \mid 0>$ do not vanish. Yet from

$$
\left\{\mathcal{Q}_{-}^{a}, \mathcal{Q}_{-}^{b \dagger}\right\}=0
$$

their norms must satisfy

$$
\left|\mathcal{Q}_{-}^{a}\right| \cdots>\left.\right|^{2}+\left|\mathcal{Q}_{-}^{a \dagger}\right| \cdots>\left.\right|^{2}=0
$$

for $a=1,2$, implying that one is negative, or both are zero. This reproduces general arguments 13 based on the absence of negative or zero norm states, which show that central charges are limited to massive representations. In the frame where only $p^{+} \neq 0$, the dynamic generators of massless supersymmetry anticommute with one another. If they do not, negative or zero-norm states are generated.

This construction does not seem to generalize to odd dimensions. We have shown this explicitly in eleven dimensions by starting with $\vec{T}$ quadratic in the 
Grassmann numbers. There a quadratic product of a Grassmann spinor transforms as 2- and 3-forms, so that to make a vector we need some c-number tensors, either a one or two-form, but the commutation with the supercharge does not have the right form.

On the other hand, the construction in $(9+1)$ dimensions is straighforward. We consider two supercharges

$$
\mathcal{Q}_{+}^{(1)}=\frac{\partial}{\partial \theta}+\frac{1}{\sqrt{2}} p^{+} \theta ; \quad \mathcal{Q}_{+}^{(2)}=\frac{\partial}{\partial \eta}+\frac{1}{\sqrt{2}} p^{+} \eta,
$$

where $\theta \sim \mathbf{8}_{s}, \quad \eta \sim \mathbf{8}_{s}^{\prime}$. We take the light-cone translation vector to be

$$
T^{i}=i p^{+} z \theta \tilde{\gamma}^{i} \eta \sim \mathbf{8}_{v}, \quad z \text { real }
$$

so that this corresponds to type IIA supersymmetry. The dynamic boosts are

$$
S^{-i}=i z \theta \tilde{\gamma}^{i} \eta-\frac{p^{j}}{p^{+}} \widehat{S}^{i j}-\frac{p^{i}}{p^{+}} S^{+-}
$$

with

$$
\widehat{S}^{i j}=S^{i j}-\frac{i}{2}\left(\theta \tilde{\gamma}^{i j} \frac{\partial}{\partial \theta}+\eta \tilde{\gamma}^{i j} \frac{\partial}{\partial \eta}\right), \quad \text { and } \quad S^{+-}=-\frac{i}{2}\left(\theta \frac{\partial}{\partial \theta}+\eta \frac{\partial}{\partial \eta}\right) \text {. }
$$

The dynamic supercharges are now

$$
\begin{aligned}
& \mathcal{Q}_{-}^{(2)}=-\frac{i p^{i}}{\sqrt{2} p^{+}} \tilde{\gamma}^{i} \mathcal{Q}_{+}^{(2)}+i \sqrt{2} z \theta \\
& \mathcal{Q}_{-}^{(1)}=-\frac{i p^{i}}{\sqrt{2} p^{+}} \tilde{\gamma}^{i} \mathcal{Q}_{+}^{(1)}-i \sqrt{2} z \eta
\end{aligned}
$$

The resulting supersymmetry algebra now includes central charge

$$
\left\{\mathcal{Q}_{+}^{(1)}, \mathcal{Q}_{-}^{(2)}\right\}=-\left\{\mathcal{Q}_{+}^{(2)}, \mathcal{Q}_{-}^{(1)}\right\}=i \sqrt{2} z .
$$

We have seen that our construction leads to supersymmetry but the representations necessarily contain negative and zero-norm states. Although it is interesting to note that central charges occur naturally whenever the lightcone translations are built out of the Grassmann variables, the representations contain negative and zero-norm states; at best they could be used as ghost compensators of some unknown theory.

\section{Conclusion}

In this paper, we considered non-zero light-cone translations to construct the continuous spin representations of both Poincaré and Super-Poincaré algebra. 
We started with the Poincaré algebra in four space-time dimensions and generalized it to higher dimensions. The light-cone translation vector, $\vec{T}$, is represented by spherical harmonics in $(d-2)$-dimensions for the light-cone little group $S O(d-2)$. We find that the states can be represented by $p^{+}$, the length $\Xi$ and $(d-3)$ number of angles of the $S O(d-2)$ spherical harmonics, and the Dynkin labels $\left(a_{1}, \cdots, a_{r}\right)$ of $S O(d-3)$ the short little group, with $r$ being the rank. There is one CSR for each representation $\left(a_{1}, \cdots, a_{r}\right)$.

If $\vec{T}$ is nilpotent, a finite number of states are generated by the light-cone boost, instead of infinite number of states, but the resulting CSR contains zero or negative norm states, and its supersymmetric extension (in four dimensions) has a central charge. The nilpotent construction of $T^{i}$ in higher dimensions is not so evident. These CSRs may be useful in conjunction with theories where the invariances force overcounting, as in ghost states in gauge theories.

Acknowledgements: Three of us(AK,PR,XX) wish to acknowledge the support in part by the US Department of Energy under grant DE-FG02-97ER41029.

\section{References}

[1] E. Wigner, Ann. Math. 40, 149 (1939).

[2] P. A. M. Dirac, Rev. Mod. Phys. 21, 392 (1949).

[3] H. Bacry and N. P. Chang, Annals of Physics 47, 407 (1968).

[4] P. Goddard, J. Goldstone, C. Rebbi, and C. B. Thorn, Nucl. Phys. B56, 109 (1973).

[5] E. Wigner in Theoretical Physics, International Atomic Energy Agency, Vienna, 1963.

[6] L. Abbott, Phys. Rev. D 13, 2291 (1976).

[7] K. Hirata, Prog. Theo. Phys. 58, 652 (1977).

[8] D. Zoller, Class. Quant. Grav. 111423 (1994).

[9] E. Witten and S. Weinberg, Phys. Lett. B96, 59 (1980);

Loyal Durand III, Phys. Rev. 128, 434 (1962);

K. Case and S. Gasiorowicz, Phys. Rev. 125, 1055 (1962).

[10] C. Aragone and S. Deser, Nuovo Cim. 57B, 33 (1980).

[11] M. A. Vasiliev, hep-th/0104246, and references therein.

[12] A. K. H. Bengtsson, I.Bengtsson, L. Brink, Nucl. Phys. B227, 41 (1983).

[13] M. Sohnius, Phys. Rep. 128, 39 (1985).

[14] R. Slansky, Phys. Rep. 79, 1 (1981). 